\title{
Editorial Comment to "A nomogram Based on a Collagen Feature Support Vector Machine for Predicting the Treatment Response to Neoadjuvant Chemoradiotherapy in Rectal Cancer Patients"
}

\author{
Akiko Yashima-Abo, MD, $\mathbf{P h D}^{1}$, Koki Otsuka, $\mathrm{MD}, \mathbf{P h D}^{2}$, and Satoshi S. Nishizuka, MD, $\mathbf{P h D}^{1} \mathbb{D}$ \\ ${ }^{1}$ Division of Biomedical Research and Development, Iwate Medical University Institute for Biomedical Sciences, Yahaba, \\ Iwate, Japan; ${ }^{2}$ Department of Surgery, Iwate Medical University School of Medicine, Yahaba, Iwate, Japan
}

In the published report by Jiang et al. the authors categorized collagen features $(\mathrm{CFs})$ in pretreatment biopsy specimens for clinicopathological analysis to predict treatment response to neoadjuvant chemoradiotherapy $(\mathrm{nCRT})^{1}$. The quantified morphological data for $\mathrm{CFs}$ were obtained using a multiphoton imaging microscope and then subsequently processed with an originally developed image processing algorithm, namely the collagen features support vector machine (CFs-SVM) classifier and its stratified derivative CFs-nomogram. Multiphoton imaging technology was used for the analysis based on nonlinear optical effects including two-photon excitation fluorescence and second harmonic generation to excite endogenous autofluorescent substances and collagen in the tissue, respectively. Based on these technological characteristics, the authors developed and validated a prediction model for treatment response to nCRT based on CFs. Although the present study is a retrospective setting, it nonetheless explores an interesting concept and supports the value of proceeding with prospective studies.

The CFs-SVM classifier produced several integrated CF categories including percentage area collagen, mean of collagen fiber straightness, and collagen cross-link density for comparison with nCRT response. For example, patients having low collagen percentage area, low mean fiber straightness and low cross-link density were classified as

(C) Society of Surgical Oncology 2021

First Received: 17 May 2021

Accepted: 8 June 2021;

Published Online: 24 June 2021

S. S. Nishizuka, MD, PhD

e-mail: snishizu@iwate-med.ac.jp low CFs-SVM group. These patient group exhibited a better chance for good response after nCRT than those with non-good response, even considering the potentially confounding factors such as age, gender, and histological type. In fact, the results were confirmed by the validation cohort. ${ }^{1}$ These findings suggest that the CFs-SVM classifier is a potential metric to inform the potential efficacy of nCRT using pretreatment biopsies prior to pretreatment evaluation of nCRT.

Crosstalk between cancer cells and stromal cells are reported to be involved in the acquired capacity for invasive growth and metastasis, which lead to treatment resistance. $^{2,3}$ Isella et al. reported that high expression levels of stem/serrated/mesenchymal subtype genes by cancer-associated fibroblasts (CAFs) is related to poor prognosis in untreated colorectal cancer (CRC), in which concurrent high-level $\mathrm{CFs}$ predicted resistance to radiotherapy in rectal cancer. ${ }^{4} \mathrm{~A}$ study by Calon et al. ${ }^{5}$ also demonstrated that the level of gene expression by CAFs was associated with poor prognosis. Meanwhile, a recent study by Ueno et al. ${ }^{6}$ validated the prognostic importance of the desmoplastic reaction (DR), which involves growth of connective tissue, in stage II colon cancer. Histopathologically, CFs, CAFs, and the morphological features of DR are part of the stromal component of a tumor and include epithelial cancer cells. ${ }^{6}$ Therefore, establishment of a sophisticated classification method to predict treatment response is scientifically reasonable.

In viewpoint of daily practice, HE staining is the current gold standard for diagnostic pathology. However, identification of CFs in tumor tissue by $\mathrm{HE}$ staining alone is difficult. Additional Masson staining is generally needed to precisely assess CFs in cancer tissue sections. Therefore, in terms of quantitative $\mathrm{CF}$ evaluation, it is clear that the CFs- 
SVM classifier model combined with a multiphoton imaging microscope is more sophisticated than a conventional optical microscope. Such computer-aided diagnosis techniques have advantages in interpreting and classifying digital data quantitatively, while effectively reduces human resources. Due to increased disease incidence, many clinical pathologists currently spend substantial amounts of time diagnosing and classifying malignant neoplasm specimens. The demand for subtyping malignant tumors based on biological and genetic properties will further increase workload for pathologists. The adoption of new diagnostic tools, such as non-labeling multiphoton imaging microscopes, when available, might offer advantages to clinical pathologists in daily practice. Nonetheless, the clinical feasibility of the CFs-SVM classifier should still be evaluated prospectively in terms of clinical utility (i.e., if diagnosis affects prognosis) in addition to considerations of the availability of multiphoton imaging microscopes at the level of daily practice. The present study offers a highly objective diagnostic capability option. If we can predict treatment response before $\mathrm{nCRT}$, then it will be beneficial to LARC patients in terms of cost-effectiveness and toxicity.

DISCLOSURE The authors declare no conflicts of interest.

\section{REFERENCES}

1. Jiang W, Li M, Tan J, Feng M, Zheng J, Chen D, et al. A nomogram based on a collagen feature support vector machine for predicting the treatment response to neoadjuvant chemoradiotherapy in rectal cancer patients. Ann Surg Oncol. 2021. https://doi. org/10.1245/s10434-021-10218-4.

2. Kalluri R, Zeisberg M. Fibroblasts in cancer. Nat Rev Cancer. 2006;6:392-401.

3. Joyce JA, Pollard JW. Microenvironmental regulation of metastasis. Nat Rev Cancer. 2009;9:239-52.

4. Isella C, Terrasi A, Bellomo SE, Petti C, Galatola G, Muratore A, et al. Stromal contribution to the colorectal cancer transcriptome. Nat Genet. 2015;47:312-9.

5. Calon A, Lonardo E, Berenguer-Llergo A, Espinet E, HernandoMomblona X, Iglesias M, et al. Stromal gene expression defines poor-prognosis subtypes in colorectal cancer. Nat Genet. 2015;47:320-9.

6. Ueno $\mathrm{H}$, Ishiguro $\mathrm{M}$, Nakatani $\mathrm{E}$, et al. Prognostic value of desmoplastic reaction characterisation in stage II colon cancer: prospective validation in a Phase 3 study (SACURA Trial). $\mathrm{Br} \mathrm{J}$ Cancer. 2021;124:1088-97.

Publisher's Note Springer Nature remains neutral with regard to jurisdictional claims in published maps and institutional affiliations. 